\title{
Reduced-dimensional models for straight-channel proton exchange membrane fuel cells
}

\author{
Gwang-Soo Kim ${ }^{a, *}$, P.C. Sui ${ }^{b}$, A.A. Shah ${ }^{c}$, Ned Djilali ${ }^{b}$ \\ a Ballard Power Systems, 9000 Glenlyon Parkway, Burnaby, BC V5J 5J8, Canada \\ ${ }^{\mathrm{b}}$ Institute for Integrated Energy Systems and Department of Mechanical Engineering, University of Victoria, PO Box 3055, STN CSC, Victoria, BC V8W 3P6, Canada \\ ${ }^{\mathrm{c}}$ Energy Technology Research Group, School of Engineering Sciences, University of Southampton, Southampton SO17 1BJ, United Kingdom
}

\section{A R T I C L E I N F O}

\section{Article history:}

Received 10 October 2009

Received in revised form

21 November 2009

Accepted 25 November 2009

Available online 1 December 2009

\section{Keywords:}

Proton exchange membrane fuel cell

CFD

Transport phenomena

Reduced-dimensionality

Efficient simulation

\begin{abstract}
A B S T R A C T
A comprehensive description of proton exchange membrane fuel cell (PEMFC) performance includes the transport phenomena, phase change and electrochemical reaction inside the several components, which possess disparate characteristics and together form a complex three-dimensional geometry. Much of the modelling work in this area has, therefore, relied on the techniques of computational fluid dynamics (CFD). The comprehensive three-dimensional (3D) approach can, however, be prohibitively time consuming. Consequently, it is not the ideal basis for a rapid screening tool that operates under a wide range of design options and operating conditions. Mathematical models and solution procedures using simplified models with reduced dimensions have been proposed to address this issue. Such approaches are computationally efficient, but no systematic study has been conducted to qualitatively or quantitatively assess the impact of the neglected dimensionality on the accuracy of the resulting model. In this paper, we compare results from a hierarchy of reduced-dimensional models to the results from a comprehensive 3D CFD model for a single, straight-channel unit cell. The quality of the simulation results from reduced-dimensional models, including the cell voltage and the distributions of current density and relative humidity, are assessed. We demonstrate that the 2+1D approach, which includes mass transport in the 2D cross-section of the channel and membrane electrode assembly and integrates along the flow channel, is optimal in terms of both efficiency and accuracy. It provides a sound basis for a simulation tool that can be used in the early stages of a unit-cell design cycle.
\end{abstract}

(c) 2009 Elsevier B.V. All rights reserved.

\section{Introduction}

In the past two decades fuel cells have emerged as a feasible alternative to conventional hydrocarbon-based power sources due to their high power density and overall low emission levels. Proton exchange membrane fuel cells (PEMFCs), which operate at relatively low temperatures, have been under development for applications over a broad range of power output in different operating environments. The structure of a PEMFC is surprisingly simple compared to incumbent technologies such as the internal combustion engines. A typical "plate-and-frame" PEMFC consists of a membrane electrode assembly (MEA) and bipolar plates that conduct electricity and provide flow pathways for the reactants and product water. The MEA is composed of an ion-exchange membrane between porous carbon electrodes (gas diffusion lay-

\footnotetext{
* Corresponding author. Current address: Intel Corp., Process Technology Modeling Group, M/S RA3-254, 2501 NW 229th Avenue, Hillsboro, OR 97124, United States. Tel.: +1 971214 0449; fax: +1 5036138950 .

E-mail address: gskim@alum.mit.edu (G.-S. Kim).
}

ers (GDL)). Despite its simple structure, the transport phenomena in a proton exchange membrane fuel cell (PEMFC) are highly convoluted. Transport of heat, charge, fluid mass and fluid momentum, together with electrochemical reactions and mass transfer between phases, are intimately related, which complicates any computational and experimental analysis. Further challenges are posed by a three-dimensional geometry with disparate length scales and by the complex morphologies of the catalyst and gas-diffusion layers, which possess quite distinct characteristics. It is natural, therefore, that a great deal of the modeling effort follows a computational fluid dynamics (CFD) approach, examples of which include [1-4]. The most comprehensive three-dimensional (3D) CFD methods, however, can be extremely time consuming, despite the significant advances made in computer hardware and software tools. This renders them unsuitable as a basis for rapid testing of design options under a broad range of operating conditions of interest-a typical industrial requirement. Mathematical models and solution procedures using simplified models with reduced dimensions have been proposed to address this issue [5-9]. Although these approaches lead to a reduction in the time cost, no systematic study has been conducted to quantitatively assess the impact of the neglected 


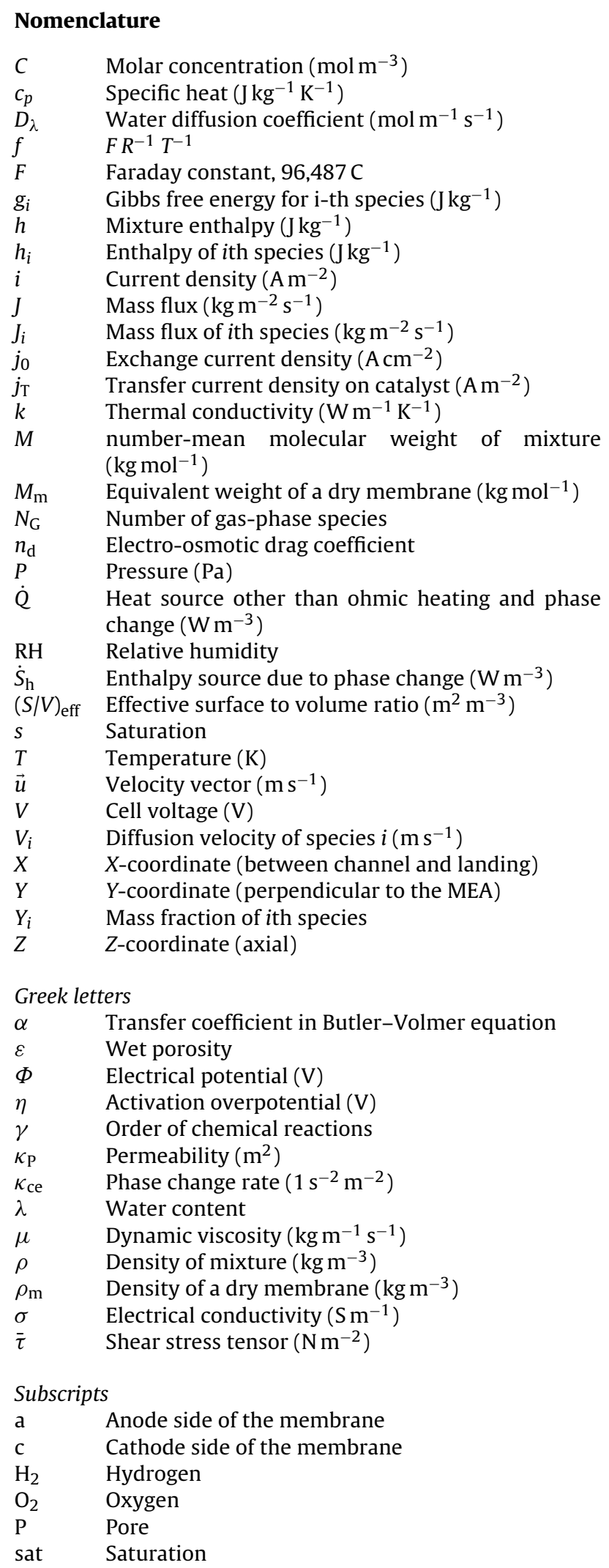

dimensionality. In this paper we employ a comprehensive 3D CFD model as the baseline for comparison with the following hierarchy of reduced-dimensional models (see Fig. 1).

- MEA models incorporating a cross-section of the gas channels and the MEA. Both 1D and 2D are discussed.

- Unit-cell models, which include a cross-section of gas channels and MEA together with an approximation of the direction downchannel: 2D and 2+1D (also termed pseudo-3D).

The aim of this paper is to propose a reduced-dimensional methodology as the basis for a code that is capable of simulating PEMFC performance rapidly and with accuracy comparable to that of a 3D CFD code. In the implementation of all the reduced-dimensional models, the same governing equations with equivalent boundary conditions and parameter values are solved, using the same solver and computer hardware. The reduceddimensional methodology developed in the present study may require modification for complex flow field geometries, e.g. serpentine channels, to take into account bend effects and cross-channel convection. Further investigations are required to deal specifically with such cases.

In the next section, the governing equations and boundary conditions are presented and the construction of each reduceddimensional models is described. In Section 3, numerical results using the hierarchy of reduced-dimensional models are compared with results from a full 3D model. The accuracy of each model is assessed and the results are discussed. Finally, conclusions are provided and key results are summarized in Section 4.

\section{Mathematical formulation and numerical method}

\subsection{The 3D CFD model}

The governing equations considered in the present study are summarized in Table 1 . These are conservation of mass, momentum, gas species, charge (electronic and ionic) and thermal energy, which are solved in the entire computational domain; more details on the formulation and implementation of the comprehensive model can be see in Sui et al. [10]. The transport of water across the membrane is described by the phenomenological model of Springer et al. [11], which includes mechanisms for diffusion and electro-osmotic drag. For simplicity, we assume a constant diffusion coefficient for water transport in the membrane. A fully two-phase flow approach based on liquid water transport in porous media is implemented in the full 3D model [12] but not in the reduced-dimensional models. Instead, the operating conditions for the baseline case are carefully chosen to prevent condensation. A commercial software package, CFD-ACE+ v. 2006, was used to perform all 3D simulations in the present study. The computational results obtained using the 3D model are employed as the "exact" solution to the transport problem, against which the reduceddimensional models are compared.

\subsection{Reduced-dimensional models}

In the reduced-dimensional models the set of governing equations discussed in Section 2.1 are also employed with at least one dimension decoupled from the others. Several such models have reported in the literature, including 1D MEA models $[13,14]$ and $1+1 \mathrm{D}$ unit-cell models $[5,7,8]$. The 1D MEA model resolves mass transport through MEA, but the variation along the channel is neglected. The $1+1 \mathrm{D}$ unit-cell model improves on the $1 \mathrm{D}$ MEA model by independently approximating variations in the reactant concentrations along the channel. Variations in the direction 


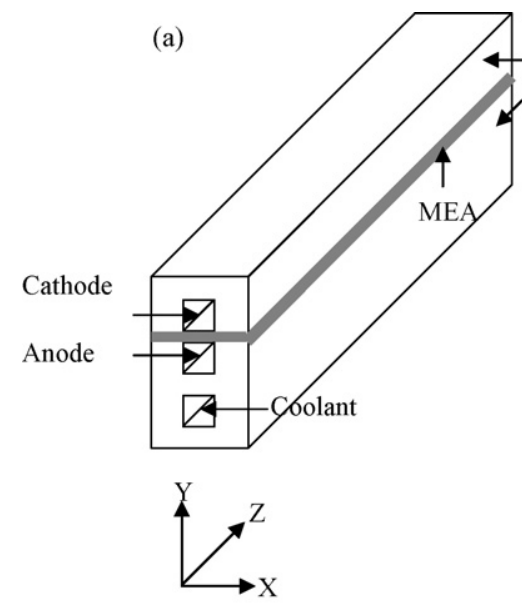

(b) Bipolar
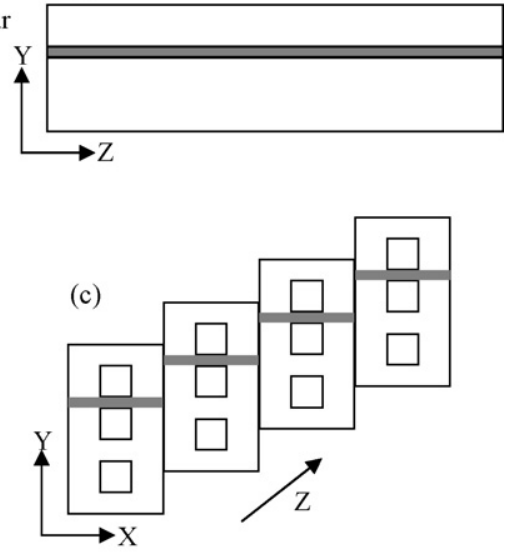

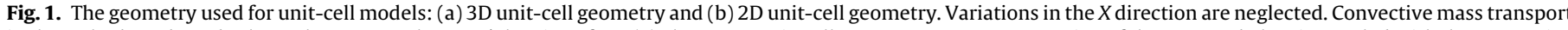

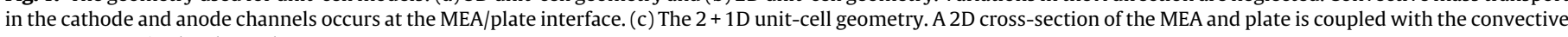
mass transport in the channel.

are, therefore, only partially resolved via an approximate conservation of mass. As such, the terminology "pseudo-2D" is often used to describe 1+1D unit-cell models. The simplicity of $1+1 \mathrm{D}$ unit-cell models allowed describing coupling between different unit cells, leading to stack model [15-17]. In the present study we develop a model that considers local, two-dimensional crosssections together with the transport along the channel. Since the computational domain for this model does not include the components outside the GDL, this approach yields a $2+1 \mathrm{D}$, or "pseudo-3D" unit-cell model.

Fig. 1 depicts the computational domain for the straight, single-channel unit-cell used for the 3D CFD calculations and reduced-dimensionality study. At the gas channel inlets, the mass flow rate and mass fractions of the gas are specified, based on a desired stoichiometric ratio and a desired dew point. Except at the inlets and outlets shown in Fig. 1, zero-flux boundary conditions for mass, temperature and potential apply at all boundary faces. Physically, these conditions imply that the system is adiabatic and that there is no leakage of reactants from the cell. The potential on the top and the bottom surfaces is varied.

Due in part to the thin-layer structure of the MEA and the imposed potential boundary conditions, it is expected that the gradient of the primary variables (concentration of gas species and potentials) are steep in the direction perpendicular to the MEA ( $Y$ coordinate). This direction is termed the 'primary direction' in the present study. The gas phase in both the anode and cathode flows in the axial direction ( $Z$-coordinate). Under normal operating conditions the reactant concentrations exhibit significant variations between the inlet and outlet, due to consumption along the flow pathway. This direction is termed the 'secondary direction' in this paper. In a number of reduced-dimensional studies, the transport in the lateral direction, i.e., $X$-coordinate, is assumed negligible, leading to the so-called 1 + 1D unit-cell model, cf. Fig. 1(b). However, the transport in this direction, called the 'tertiary direction', depends on the channel spacing [13], invalidating this assumption in some cases. Nevertheless, the $1+1 \mathrm{D}$ approximation greatly simplifies the numerical description, and often algebraic manipulation is feasible $[7,8]$. In the present study we extend the $1+1 \mathrm{D}$ unit-cell model to include the transport in the tertiary direction, cf. Fig. 1(c), thereby taking account of the distribution in the $X Y$ plane at a lower computational cost compared to full 3D model. It is noted that the $1+1 \mathrm{D}$ model simplifies the transport phenomena outside the MEA. Effects such as convection in the gas channel are, therefore, treated in a simplified form. A similar treatment can be used to simplify the 3D simulation. For mass and charge transport the high aspect ratio of the PEMFC suggests that diffusion in $Z$ direction (down the channel) is negligible compared to that in the $X-Y$ plane, while convective mass transport in this direction is dominant. Moreover, fluid flow in the channel can be approximated as a plug flow with an averaged velocity. With this approximation, each of the cross-sections

Table 1

Conservation equations solved in the comprehensive numerical simulation.

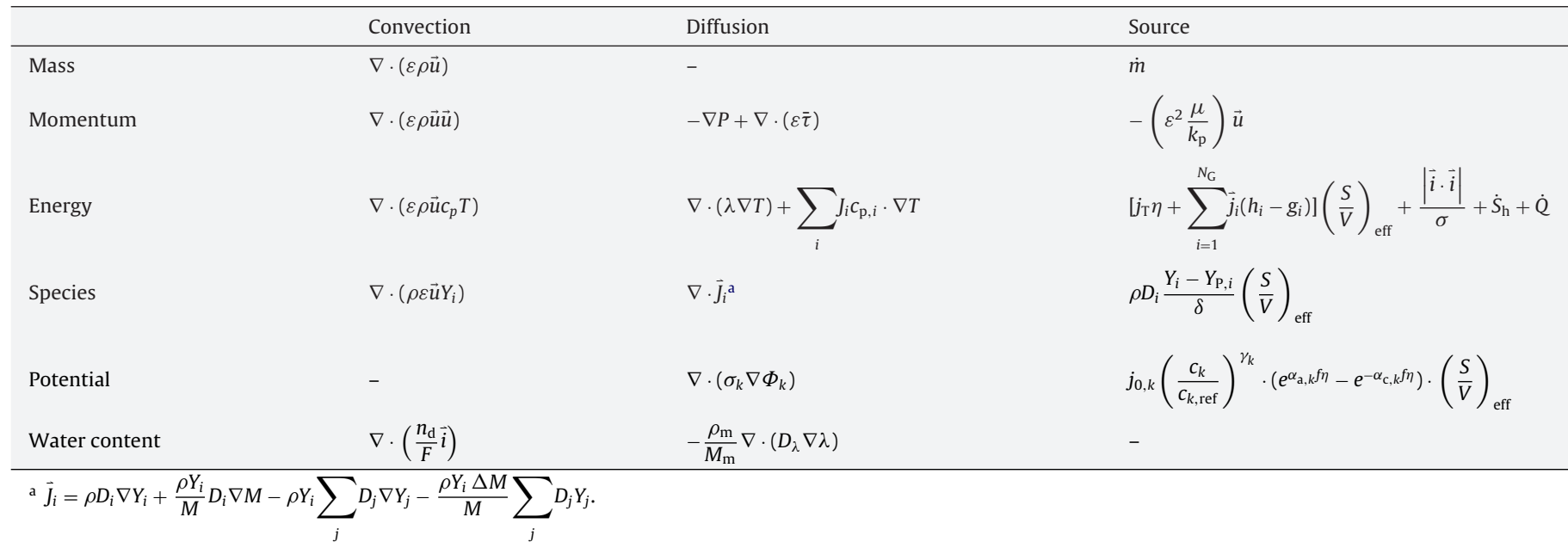




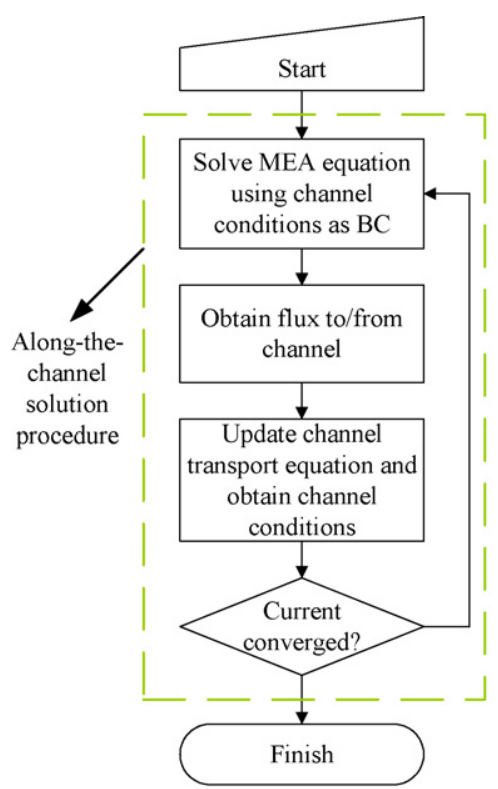

(a)

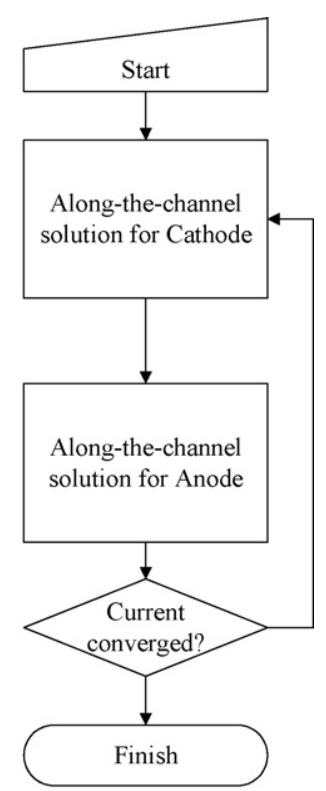

(b)
Fig. 2. Flow chart depicting the code methodology: (a) co-flow and (b) counter-flow.

is coupled through convective transport in the channel. This is the so-called pseudo-3D or 2+1D unit-cell model.

\subsection{Solution procedure}

The same set of governing equations is employed for the $3 \mathrm{D}$ and the reduced-dimensional (2D and pseudo-3D) unit-cell simulations. The solution procedure for the reduced-dimensional approach is described in Fig. 2. In the co-flow case, the anode and cathode flows are treated in the same fashion: starting from the inlet, gas concentrations are used as channel boundary conditions, together with an applied cell voltage, cf. Fig. 2(a). Once the boundary-value problem of the MEA transport is solved, the local current density and the flux from the MEA to the channel are recorded. A plug-flow model is used to predict the channel concentration at the next nodal point. This procedure is carried out along the entire length of the channel in the downstream direction. For the counter-flow case, back-and-forth shooting is required since the anode and cathode flows are in opposite directions. In the forward shooting, cf. Fig. 2(b), the anode concentration profile is fixed and the current distribution is calculated as in the co-flow case. Once the end of the channel is reached, the cathode concentration profile is fixed, and the anode concentration profile and current distribution are updated while marching in the backward direction. This iteration is repeated until the residual of the current distribution falls below a specified tolerance. The transport equations for the 1D and 2D MEA cross-sections of the 2D and pseudo-3D models, respectively, were implemented in the commercial code COMSOL Multiphysics 3.1. The convective mass-transport equation in the channel was solved using MATLAB.

\section{Results and discussion}

Numerical results obtained from different reduced-dimensional models are compared with the baseline case, for which the parameters are shown in Table 2. These transport properties and model parameters were validated with experimental data for the 3D unitcell model. Further information regarding the determination of the baseline case and experimental validation can be found in [18]. The operating conditions for the baseline case are as follows. Air and hydrogen are both humidified at a dew point of $40^{\circ} \mathrm{C}$ for the cathode and anode, respectively. The inlet temperature for all fluid channels is set to be $343 \mathrm{~K}$. The flow rates of air and hydrogen at the inlet are set to reach a stoichiometric ratio of 5 based on a current density of $1 \mathrm{~A} \mathrm{~cm}^{-2}$. With the low relative humidity (RH) and high stoichiometric ratio for the inlet gas mixtures, no liquid water was found in the entire domain under the current density conditions tested in the present study. The low RH operating conditions are in fact the trend of fuel cell application in order to eliminate or reduce parasitic power required for humidification and the effects of liquid water flooding. However, this comes at the cost of reduced membrane conductivity and an increased rate of chemical membrane degradation $[14,19]$.

Table 2

Summary of properties and parameters used for the baseline calculation.

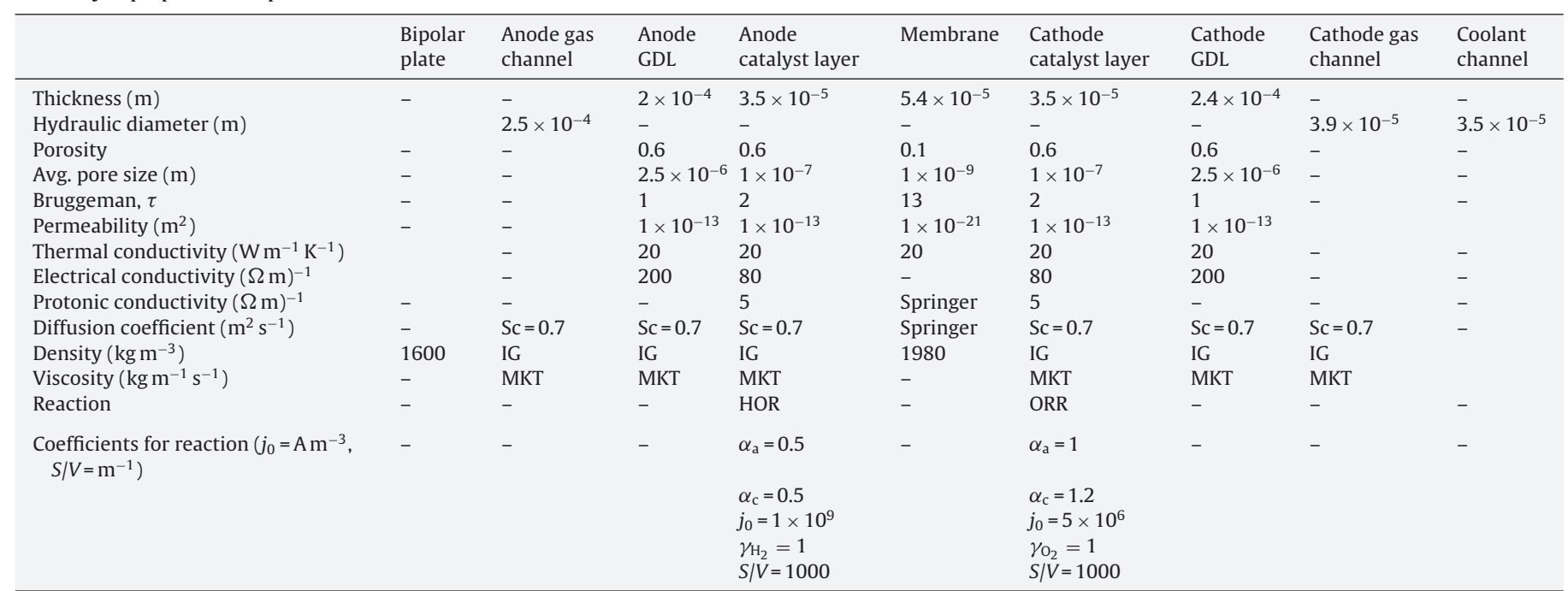

IG: ideal gas [20].

MKT: mixed kinetic theory [20].

HOR: hydrogen oxidation reaction.

ORR: oxygen reduction reaction.

Sc: Schmidt number. 


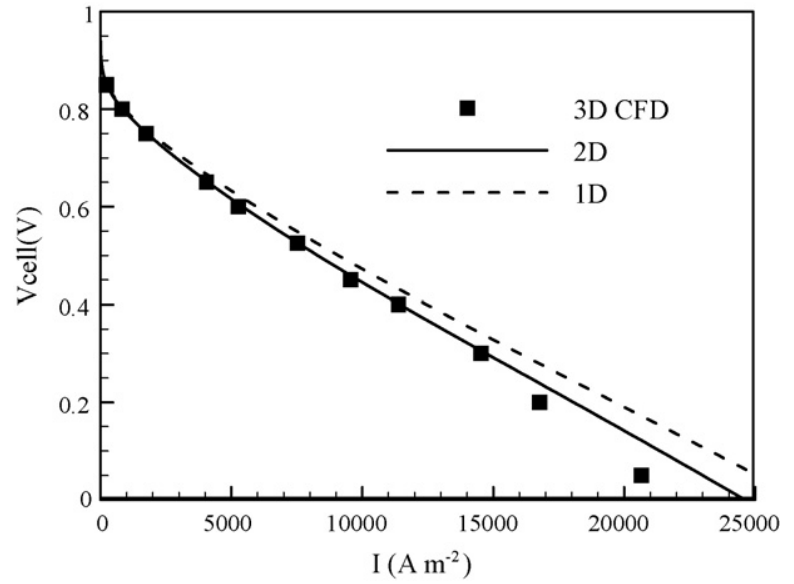

Fig. 3. A comparison between the $1 \mathrm{D}, 2 \mathrm{D}$ and $3 \mathrm{D}$ results for the baseline conditions.

In the next sections, we will demonstrate how the comprehensive 3D unit-cell model can be simplified to $2+1 \mathrm{D}$ and $2 \mathrm{D}$ equivalents. At each step, we will discuss the justifications for the simplification and the various sources of discrepancy against the baseline case.

\subsection{Effects of dimensionality in the MEA model}

In order to assess the validity of the numerical implementation, polarization curves were constructed using 1D and 2D MEA models; these are shown in Fig. 3. The strategy behind the reduceddimensional was described above. An identical set of governing equations (with identical parameters) is solved in both the 1D and 2D MEA models. The results were compared with those from a fully 3D unit-cell model, assuming short channels and a high stoichiometry to minimize variations in the axial $(Z)$ direction. The $1 \mathrm{D}$ and 2D models perform well for current densities in the range $0-1 \mathrm{~A} \mathrm{~cm}^{-2}$. For current densities greater than $1 \mathrm{~A} \mathrm{~cm}^{-2}$, the polarization curves begin to diverge from the 3D result. For the 1D MEA model, since it does not consider the rib-channel geometry, the additional ohmic losses due to the presence of the rib (see [21]) are zero, resulting in a higher predicted cell voltage. For the 2D MEA model, ohmic losses due to the presence of the rib are considered but the cell potentials are still over-predicted. In the 3D model results, it is found that liquid water forms at high current density conditions. Because the transport of liquid water is not considered in the 2D MEA model, the additional mass-transport resistance due to liquid water accumulation is not captured, thus a higher cell voltage, compared to the 3D model, is predicted.

Figs. 4 and 5 show the polarization curves and the "normalized water balance" predicted by the 1D and 2D MEA models at different $\mathrm{RH}$ values. The normalized water balance $\alpha$ is defined as

$\alpha \equiv \frac{\left[\dot{m}_{\mathrm{w}, \mathrm{IN}}-\dot{m}_{\mathrm{w}, \mathrm{OUT}}\right]_{\text {anode }}}{I / 2 F}$

where $\dot{m}_{\mathrm{w}, \mathrm{IN}}$ and $\dot{m}_{\mathrm{w}, \mathrm{OUT}}$ are mass flow rate of water at the cell inlet and outlet respectively, and $I$ is current density. Parameter $\alpha$ is a measure of the competition between electro-osmotic drag driven by potential gradients and back diffusion driven by concentration gradients. A positive value of $\alpha$ means a net water transfer from the anode to the cathode through the membrane. In Fig. 4(a), although the polarization curves appear to be identical, there are significant differences in the predicted values of $\alpha$, as seen from Fig. 4(b). The $\alpha$ value from the 1D MEA model is greater at high current density and smaller at low current density, compared to the 2D MEA model, suggesting differences in the water mass-transport behavior. This result highlights the importance of dimensionality in MEA models.
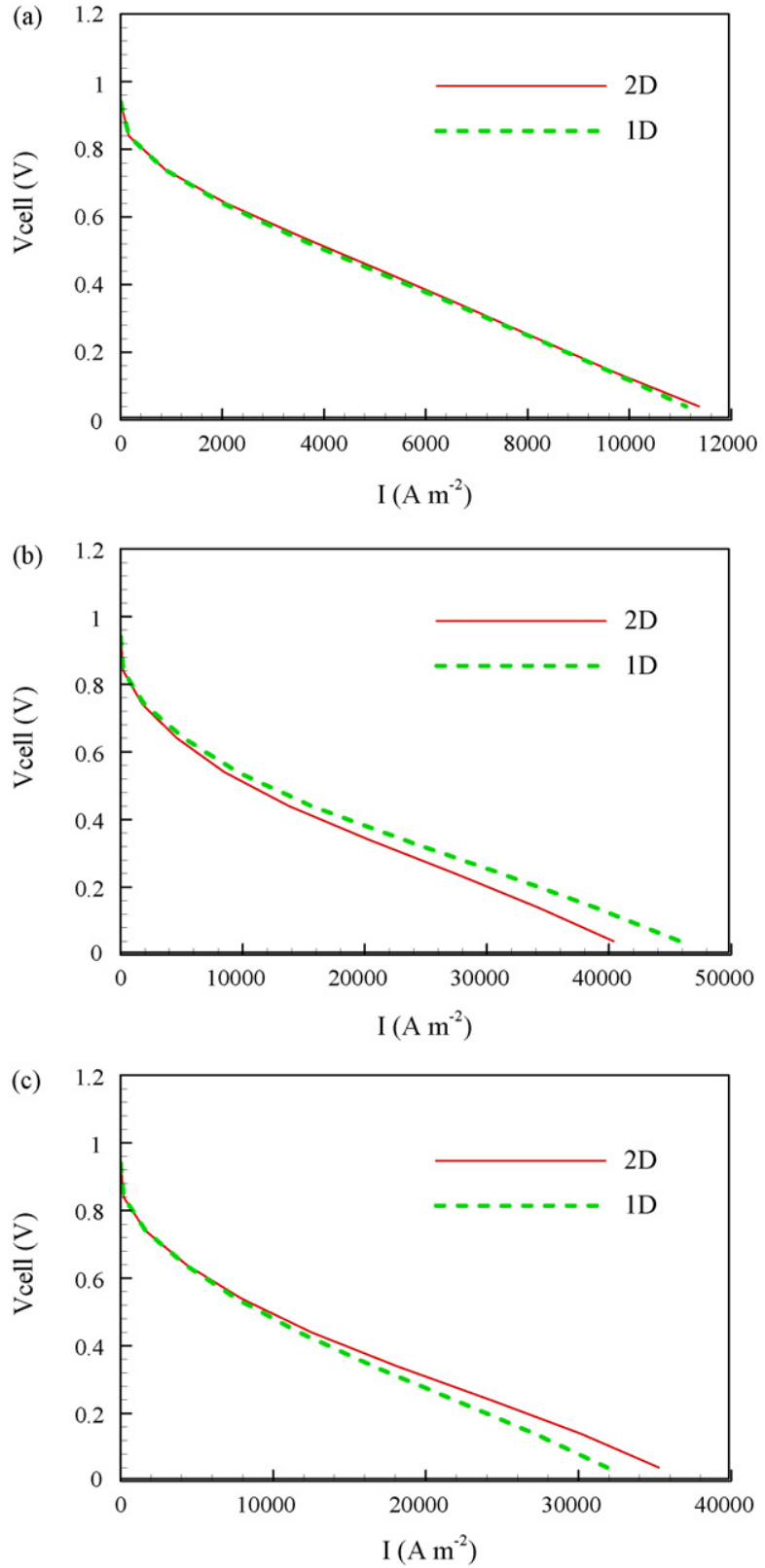

Fig. 4. (a) The polarization curve for $\mathrm{RHc}=\mathrm{RHa}=0.1$; (b) the polarization curve at $\mathrm{RHc}=0.1, \mathrm{RHa}=0.9$; (c) the Polarization curve for $\mathrm{RHc}=0.9, \mathrm{RHa}=0.1$. The other parameter values are given in Table 2 .

The remaining curves of Fig. 5(a)-(c) at different values of RH reveal similar trends.

The difference between the 1D and 2D cases lies in the channel geometries assumed. The 1D MEA model neglects variations in the cross-channel direction and effectively assumes that the gas channels are in full contact with the MEA interface. The 2D MEA model considers that only a fraction of the MEA is exposed to the channel, since the channel landing area supports the plates. The 1D simplification of the geometry does not capture the mass transport of water as effectively as the more realistic 2D MEA model. The impact of the lower mass-transport resistance predicted by the 1D model depends on the operating conditions simulated. At high levels of humidity, it is advantageous to humidify the membrane at low current densities and to expel product water effectively to the channels in order to prevent water condensation. The situation is less straightforward at low $\mathrm{RH}$ since the water required to humidify the membrane can originate from two sources: the channel (external 

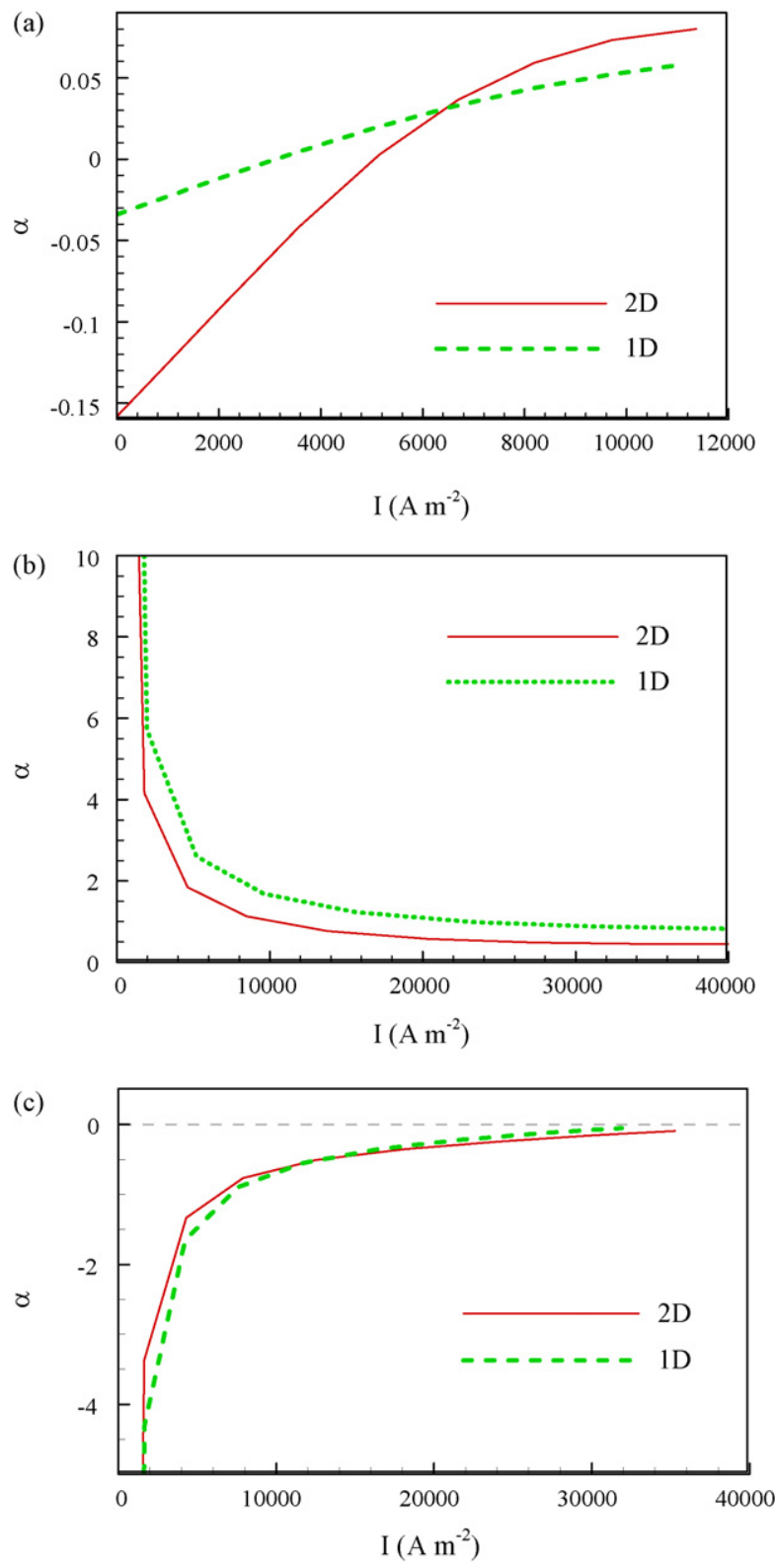

Fig. 5. (a) The normalized water balance $(\alpha)$ for $\mathrm{RHc}=\mathrm{RHa}=0.1$; (b) $\alpha$ for $\mathrm{RHc}=0.1$, $\mathrm{RHa}=0.9$; (c) $\alpha$ at $\mathrm{RHc}=0.9, \mathrm{RHa}=0.1$. The other parameter values are given in Table 2.

supply) and electrochemical reaction in the cathode catalyst layer. A lower mass-transport resistance implies that product water is more effectively transported to the channel, reducing the volume of water available to humidify the membrane, potentially leading to deterioration in performance (voltage loss).

\subsection{Comparison between the $2+1 D$ and $3 D$ unit-cell models}

The first task of the validation is to assess the quality of the $2+1 \mathrm{D}$ results compared to the $3 \mathrm{D}$ model results. Both co- and counterflow cases are shown in Figs. 6 and 7. With a given total current, the current density distribution and $\mathrm{RH}$ profile are compared. For the 3D model, the current density results are obtained by integrating the current density distribution over a constant area along the axial direction. The $\mathrm{RH}$ is averaged in the gas channel over the same axial distance. Figs. 6(a) and 7(a) show the current density distribution and $\mathrm{RH}$ profiles in the 3D and 2+1D unit-cell models for a co-flow case with low inlet RH. From the comparison, one can see
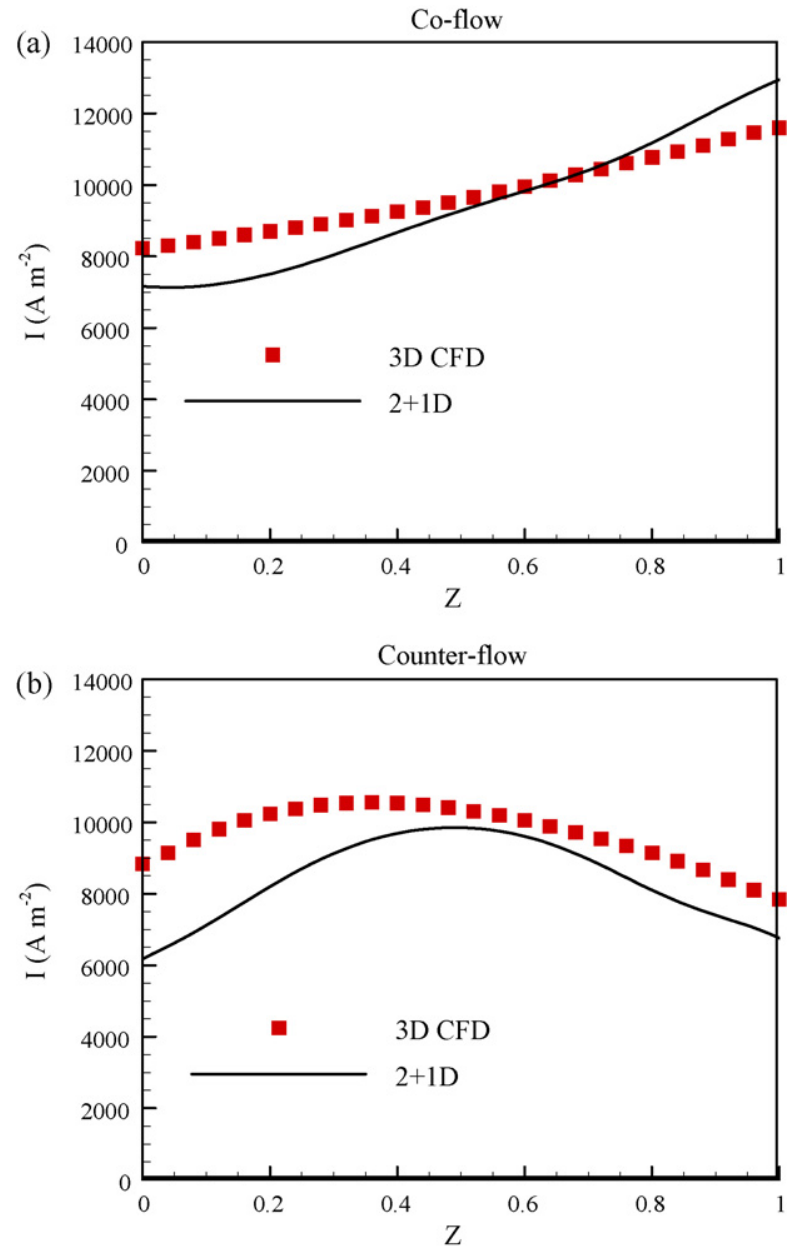

Fig. 6. A comparison of the pseudo-3D and 3D CFD results: the current density $(I)$ for (a) co-flow and (b) counter-flow. The other parameter values are given in Table 2. $\mathrm{Z}$-coordinate is axial coordinate normalized with actual channel length $(0.6 \mathrm{~m})$.

that the current distribution and $\mathrm{RH}$ profiles in the 3D and $2+1 \mathrm{D}$ cases qualitatively match. Fig. 6(a) demonstrates that the current density steadily increases from the inlet to the outlet for the co-flow case, since the membrane is progressively hydrated by the water created in the catalyst layer (as indicated by the increasing $\mathrm{RH}$ in the cathode/anode channels).

Switching the channel configuration from co-flow to counterflow alters the mass-transport behavior and the profiles of current density and RH. As shown in Figs. 6(b) and 7(b), the counter-flow case exhibits quite different current density and RH distributions from the co-flow simulations. The $\mathrm{RH}$ in the cathode increases near the inlet and remains constant at the outlet, as shown in Fig. 7(b). The flat RH profile of the cathode near the outlet indicates that the loss of water to the anode via diffusion is balanced by the gain of water from the anode via drag. In contrast to the cathode side, the $\mathrm{RH}$ of the anode increases near the inlet and decreases near the outlet. A large concentration gradient of water vapor near the inlet ensures that diffusion is the dominating transport mechanism, driving water from the cathode to the anode. When the RH of the anode becomes larger than the $\mathrm{RH}$ of the cathode, both diffusion and electro-osmotic drag are in the same direction and thus both drive water from the anode to the cathode, eventually decreasing anode RH near the outlet. Based on the RH behavior, it can be inferred that the membrane humidity will increase down the channel from the inlet ( $Z$ direction) to a maximum value in the middle portion of the unit cell. The humidity decreases along the remain- 

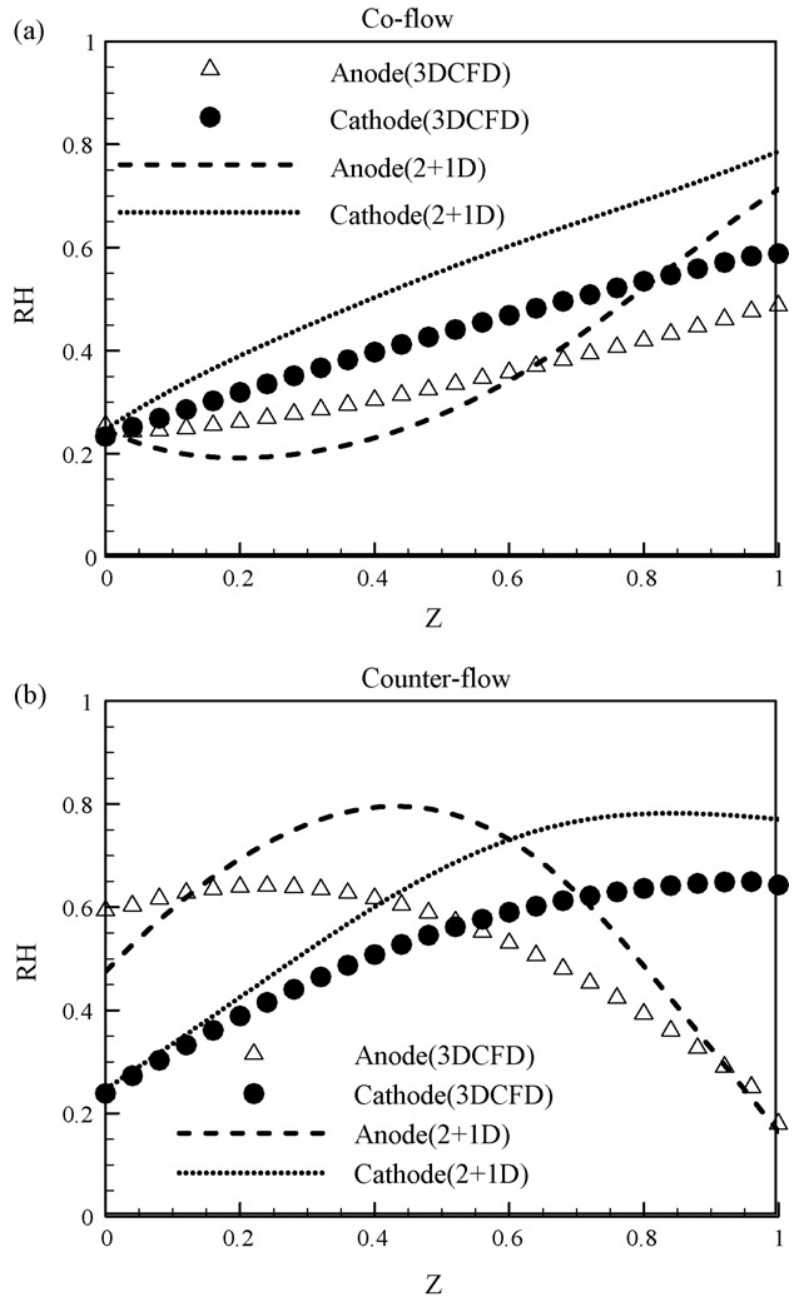

Fig. 7. A comparison of the pseudo-3D and 3D CFD results: the relative humidity $(\mathrm{RH})$ for (a) co-flow and (b) counter-flow. The other parameter values are given in Table 2 .

ing portion of the channel. Influenced largely by the membrane water content, the current density follows the same trend, with a maximum as shown in Fig. 6(b).

Thus far, simulation results from both 3D and 2+1D unit-cell models have shown that the current density distribution is heavily influenced by the RH distribution. The RH profiles, in turn, can be explained by mass transport in the MEA, diffusion and electro-osmotic drag in the membrane, and transport in the channel by convection. In the calculation discussed in this section, mass-transport resistance between the channel and the MEA was incorporated using a Sherwood number [22]. With a Sherwood number of 2 , the profiles are in good qualitative agreement with the $3 \mathrm{D}$ results without parameter calibration. This illustrates that the mass-transport behavior of the 3D model is well approximated by the $2+1 \mathrm{D}$ unit-cell approach.

Our next step is to investigate whether further simplifications can be made in relation to the mass-transport problem in the plane representing a cross-section of the MEA, thereby increasing the simulation throughput time. Several simplified models, such as the $1+1 \mathrm{D}$ unit-cell model, have been reported in the literature $[7,8]$. To the best of our knowledge, a systematic comparison of these models to higher dimensional models has not been made. Understanding the differences between the various model-reduction approaches and calculating the magnitudes of the errors associated with each, facilitates the process of selecting a model for a given purpose. In the
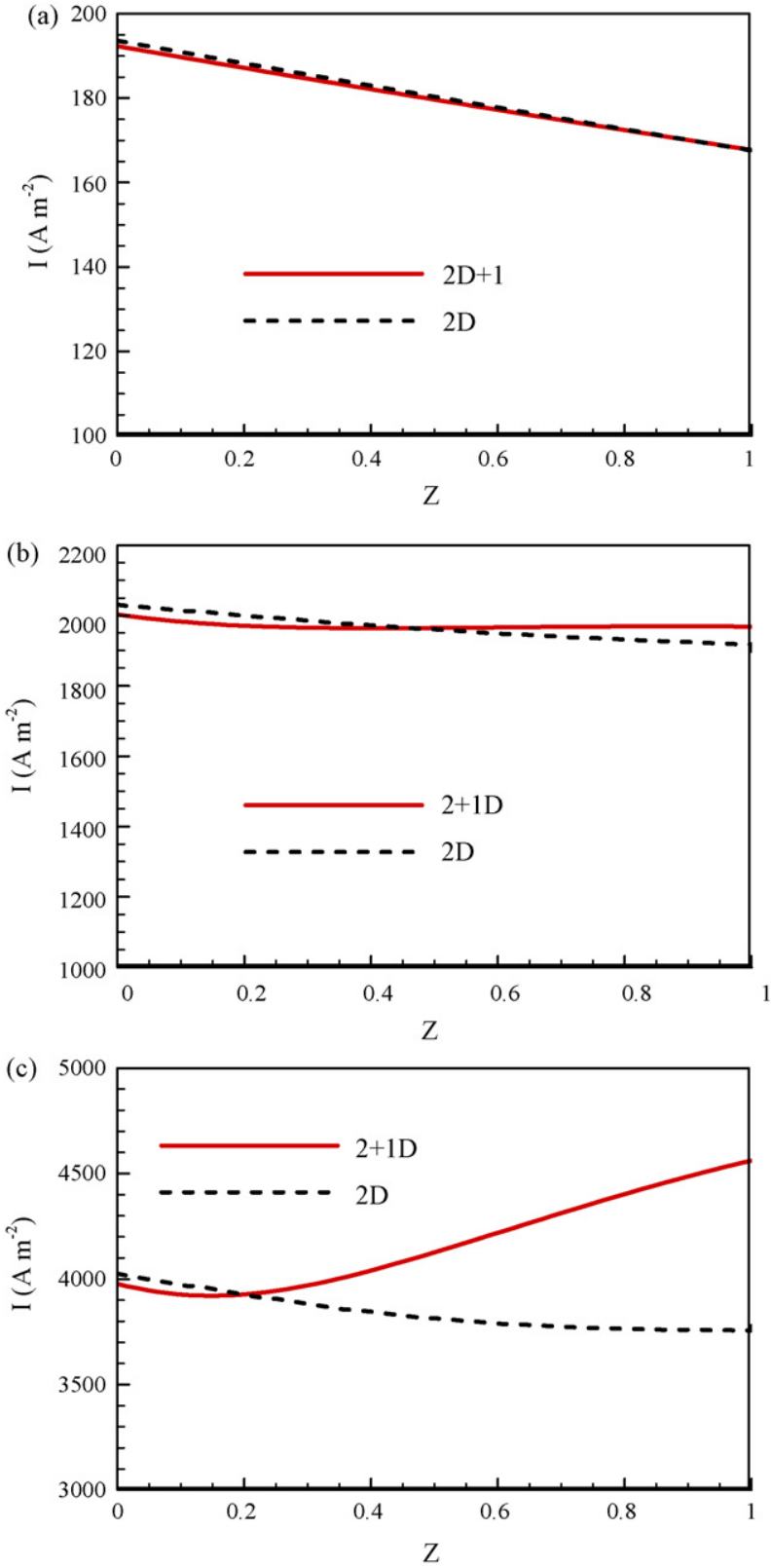

Fig. 8. A comparison between $2+1 \mathrm{D}$ and $2 \mathrm{D}$ unit-cell models: (a) $V=0.84 \mathrm{~V}$, (b) $V=0.7 \mathrm{~V}$ and (c) $V=0.6 \mathrm{~V}$. The other parameter values are given in Table 2 .

following section, models that employ these simplified geometries are evaluated.

\subsection{Comparison between the $2 D$ and $2+1 D$ unit-cell models}

The examples in Section 3.1 show that the 1D MEA model under-predicts mass-transport resistance. The 1D MEA model can be extended to include convective mass transport in the channel, leading to the $1+1 \mathrm{D}$ unit-cell model. In this work, instead of the $1+1 \mathrm{D}$ model, a true 2D model was constructed, which solves the transport problem in the axial direction. Since the transport in the MEA along the axial direction is relatively weak [7,8] compared to the direction perpendicular to the MEA, it is expected that the $1+1 \mathrm{D}$ and 2D unit-cell models will result in close agreement.

The simulation results of the $2 \mathrm{D}$ and the $2+1 \mathrm{D}$ unit-cell models are compared in Fig. 8. In the present study, the mass-transport resistance between the MEA and gas channels was ignored. Results at a low inlet $\mathrm{RH}$ and three different applied cell voltages, 0.84 , 
0.7 , and $0.6 \mathrm{~V}$, are shown for comparison. At a high cell voltage $(0.84 \mathrm{~V})$, the agreement between the $2+1 \mathrm{D}$ and the $2 \mathrm{D}$ unit-cell model is good, as demonstrated in Fig. 8(a). At this high cell voltage value, which corresponds to a low current density, mass-transport effects are minor due to the slow reaction rate. At a lower voltage, discrepancies in the current density distribution between the two models become more noticeable as mass (particularly water) transport effects begin to dominate. At low cell voltage $(0.6 \mathrm{~V})$, it is apparent that the current density at the outlet of the $2+1 \mathrm{D}$ unit-cell geometry is higher than the corresponding current density in the 2D unit-cell model, as shown in Fig. 8(b) and (c). In the $2 \mathrm{D}$ model, the current density is almost uniform since water readily escapes from the MEA to the channel, removing a source of humidification for the membrane in the direction down the channel. In the 2+1D unit-cell model, in contrast, the current density increases down the channel due to the additional humidification of the MEA from a higher mass-transport resistance for water.

It is noted that the $2 \mathrm{D}$ results does not match the $2+1 \mathrm{D}$ results since the approximation of the 2D MEA by a 1D MEA under-predicts the mass-transport resistance. To compensate for this shortcoming, an additional mass-transport resistance may be introduced at the boundary between the GDL and the gas channel in the form of an effective Sherwood number. This mass-transport resistance needs to be determined by calibration against experimental data or a by using a higher dimensional model.

\subsection{Calibration of reduced-dimensional models: comparison of the $2 D$ and $3 D$ unit-cell models}

To construct the entire polarization curve, the computational time for the 2D unit-cell model is typically less than a few minutes, but generally the results do not match those of the 3D unit-cell model. However, it is possible to fit the 2D model results to those from the 3D model by tuning the mass-transport coefficient (or Sherwood number, Sh) at the interface between the MEA and channel. The 2+1D model fully resolves the cross-channel effects and includes convective mass transport in the channels. A typical computational time is $30 \mathrm{~min}$ on an Intel Pentium $4^{\mathrm{TM}}$ processor $(2 \mathrm{GHz})$ and the results generally match very closely those of the 3D models, without the need of fitting parameters.

Fig. 9 shows a comparison between the 2D and 3D unit-cell model results. The plots indicate the sensitivity of the current density distribution to the choice of Sh in both the co-flow (Fig. 9(a)) and counter-flow (Fig. 9(b)) cases. With a typical value of $\mathrm{Sh}=2$ (laminar conditions), the inlet and outlet current density values are over-predicted for the co-flow case. With a very large $S h$, in this case 100 , a similar trend is observed. For the counter-flow case, the same value of Sh generates a current density distribution that is qualitatively different from the 3D unit-cell result; in the latter, the current density reaches a maximum in the first half of the cell, but with $S h=2$, the maximum lies in $Z=0.8$. With $S h=100$, the current density does not reach an interior maximum, but monotonically increases from the inlet to the outlet.

In order to obtain agreement with the 3D unit-cell result, the Sherwood number and the voltage were adjusted until the current density distributions matched. This iterative fitting procedure is inherently time consuming and is not well suited to the study of a large number of cases. Figs. 9(a) and 10(a) show the current density and $\mathrm{RH}$ profile with $\mathrm{Sh}=0.34$ and $V=0.47 \mathrm{~V}$ in the co-flow operation, respectively. The agreement between the $2 \mathrm{D}$ and $3 \mathrm{D}$ cases is surprisingly good. However, the Sherwood number had to be fitted again to match $3 \mathrm{D}$ result in the counter-flow operation (see Figs. 9(b) and 10(b)), now taking the value $S h=0.46$. It is worth noting that the fitted Sherwood numbers for co- and counter-flow are smaller than the realistic value of 2 (in addition to being differ-
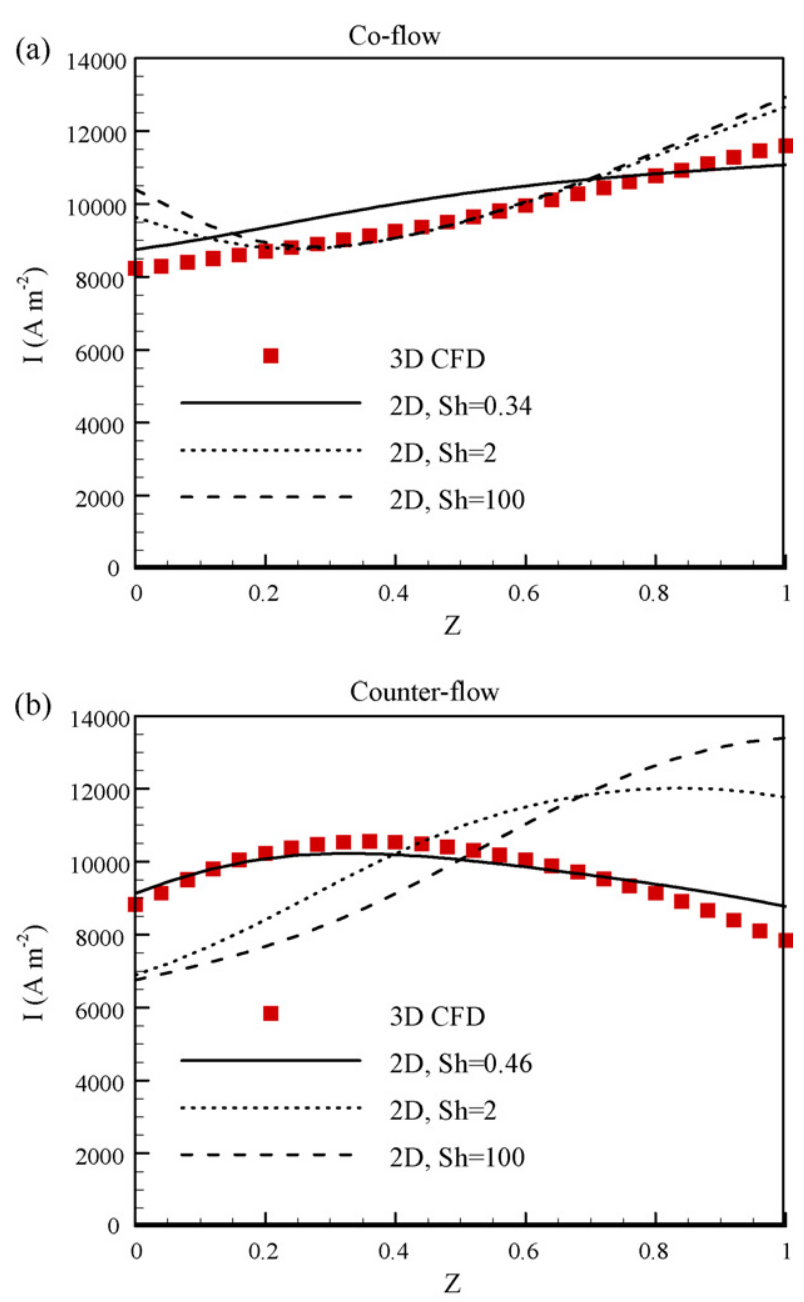

Fig. 9. A comparison of the $2 \mathrm{D}$ and $3 \mathrm{D}$ CFD results: the current density $(I)$ for (a) co-flow and (b) counter-flow. The other parameter values are given in Table 2.

ent for the co- and counter-flow cases). It would appear that only a small value of the Sherwood number is required to account for the neglected mass-transport resistance.

Thus far, we have demonstrated how the comprehensive 3D CFD model can be systematically reduced to a lower-dimensional model with additional fitting parameters. Since convection is the dominant mass-transport mechanism in the channel, the first simplification to the 2+1D model was well justified and closely reproduced the $3 \mathrm{D}$ results. Further simplification of the $2+1 \mathrm{D}$ model to form the $2 \mathrm{D}$ model resulted in a greater discrepancy since the 2D mass transport in the MEA can be approximated as $1 \mathrm{D}$ only in a narrow window of operating conditions. Beyond this narrow range, the original set of parameters does not reproduce the multidimensional phenomena without additional fitting parameters, such as a Sherwood number.

Although model discrepancies associated with the dimensionality are considered in this work, in practice, the parameters and models themselves contain errors. If calibration of the model parameters is inevitable, the reduced-dimensional model is more efficient in fitting model parameters, especially if the fitting requires multiple numerical solutions. In fact, parameters can be fitted in the reduced model and then used for higher dimensional models. Such calibrated models can be useful for designing a process controller, where the simulation run time is important and the operating conditions remain in the neighborhood of a predefined set of point. Calibration models, however, have limited applica- 

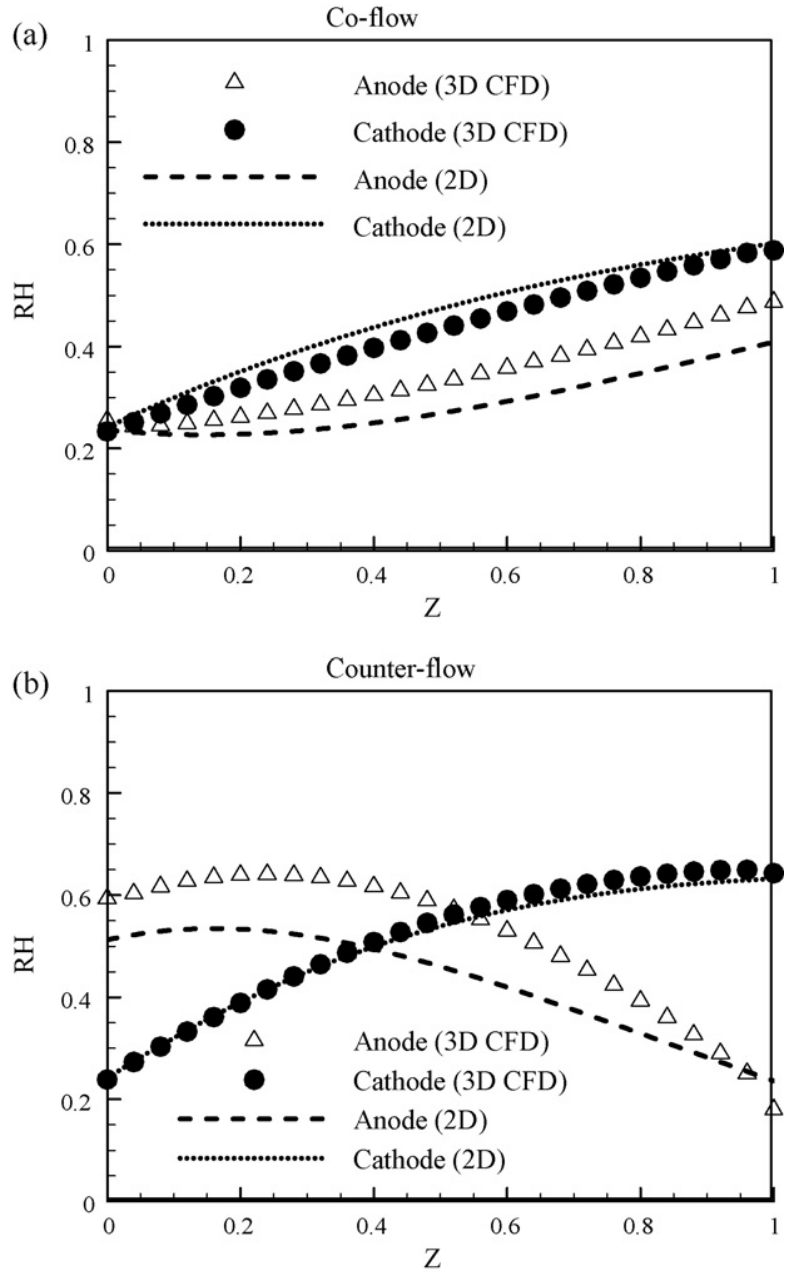

Fig. 10. A comparison of the $2 \mathrm{D}$ and $3 \mathrm{D}$ CFD results: the relative humidity $(\mathrm{RH})$ for (a) co-flow and (b) counter-flow. The other parameter values are given in Table 2.

bility for process optimization and for designing fuel cells if the model parameters are affected by the operating condition or fuel cell design.

It has been emphasized in this work that the water vapor concentration dramatically influences the performance of the PEMFC. Increased humidification of the MEA improves fuel cell performance if membrane resistance is a limiting factor. This suggests that fuel cell performance at low relative humidity can be improved if one can selectively increase the mass-transport resistance of the water, without increasing the mass-transport resistance of the oxygen and the hydrogen. However, an excessive rise in water vapor concentration needs to be avoided in order to prevent water condensation, which can further restrict oxygen and hydrogen mass transport.

\section{Conclusions}

In this paper, reduced-dimensional models for a PEM fuel cell were investigated through a series of simulations. The numerical results were compared against those from a full 3D computational model. The differences between the 2D and 2+1D unit-cell model results indicate that the coupling between mass, heat and charge transport is important, particularly in the cross-channel direction. When mass transport of water is not captured adequately, the distribution of the hydration level along the channel is strongly affected, resulting in skewed current distributions. It is encouraging that a $2 \mathrm{D}$ unit-cell model can be calibrated to approximate the behavior in a real fuel cell geometry, although this comes at the cost of a rather time-consuming fitting procedure, which represents a critical limitation. Moreover, the value of the Sherwood number, which was introduced to achieve a match with the 3D results, is likely to depend on the given set of operating conditions and configuration.

The 2+1D unit-cell model reported in this paper is far less time consuming than the comprehensive 3D unit-cell model and, crucially, achieves a comparable level of accuracy. It does not suffer from the problems encountered in the $2 \mathrm{D}$ case. The implication of this result is that the $2+1 \mathrm{D}$ approach is (currently) optimal as a basis for a numerical code to screen MEA and unit cell designs, particularly in the early stages of a design cycle.

The analysis in this paper was restricted to the straight-channel configuration. For bipolar plates with complex flow field geometries, such as serpentine channels, bend effects and cross-channel convective flow through the GDL may introduce further complications. A separate analysis is required to deal specifically with such cases.

\section{Acknowledgements}

ND and PCS acknowledge the financial support provided by the MITACS Network of Centres of Excellence and Ballard Power Systems. The authors wish to acknowledge the insightful inputs of John Kenna and Brian Wetton. ND and PCS are grateful for software licenses of CFD-ACE+ from ESI-CFD.

\section{References}

[1] V. Gurau, H.T. Liu, S. Kakac, Two-dimensional model for proton exchange membrane fuel cells, AIChE Journal 44 (11) (1998) 2410-2422.

[2] T. Berning, D.M. Lu, N. Djilali, Three-dimensional computational analysis of transport phenomena in a PEM fuel cell, Journal of Power Sources 106 (1-2) (2002) 284-294.

[3] H. Ju, C.Y. Wang, Experimental validation of a PEM fuel cell model by current distribution data, Journal of the Electrochemical Society 151 (11) (2004) A1954-A1960.

[4] S. Dutta, S. Shimpalee, J.W. Van Zee, Three-dimensional numerical simulation of straight channel PEM fuel cells, Journal of Applied Electrochemistry 30 (2) (2000) 135-146.

[5] J.S. Yi, T.V. Nguyen, An along-the-channel model for proton exchange membrane fuel cells, Journal of the Electrochemical Society 145 (4) (1998) 1149-1159.

[6] A.A. Kulikovsky, Quasi-3D modeling of water transport in polymer electrolyte fuel cells, Journal of the Electrochemical Society 150 (11) (2003) A1432-A1439.

[7] A.A. Kulikovsky, 1D + 1D model of a DMFC: localized solutions and mixedpotential, Electrochemistry Communications 6 (12) (2004) 1259-1265.

[8] P. Berg, K. Promislow, J. St-Pierre, J. Stumper, B. Wetton, Water management in PEM fuel cells, Journal of the Electrochemical Society 151 (3) (2004) A341-A353.

[9] S.A. Freunberger, M. Santis, I.A. Schneider, A. Wokaun, F.N. Buchi, In-plane effects in large-scale PEMFCs-model formulation and validation, Journal of the Electrochemical Society 153 (2) (2006) A396-A405.

[10] P.C. Sui, S. Kumar, N. Djilali, Advanced computational tools for PEM fuel cell design. Part 1. Development and base case simulations, Journal of Power Sources 180 (1) (2008) 410-422.

[11] T.E. Springer, T.A. Zawodzinski, S. Gottesfeld, Polymer electrolyte fuel-cell model, Journal of the Electrochemical Society 138 (8) (1991) 2334-2342.

[12] S. Mazumder, J.V. Cole, Rigorous 3-D mathematical modeling of PEM fuel cells. II. Model predictions with liquid water transport, Journal of the Electrochemical Society 150 (11) (2003) A1510-A1517

[13] D.M. Bernardi, M.W. Verbrugge, A mathematical-model of the solid-polymerelectrolyte fuel-cell, Journal of the Electrochemical Society 139 (9) (1992) 2477-2491.

[14] A.A. Shah, G.-S. Kim, W. Gervais, A Young K. Promislow, J. Li, S. Ye, The effects of water and microstructure on the performance of polymer electrolyte fuel cells, Journal of Power Sources 160 (2) (2006) 1251-1268.

15] G.-S. Kim, J. St-Pierre, K. Promislow, B. Wetton, Electrical coupling in proton exchange membrane fuel cell stacks, Journal of Power Sources 152 (1) (2005) 210-217.

[16] G.-S. Kim, P. Chang, J. St-Pierre, Thermal Coupling Phenomena in PEMFC Experimental and Modeling Approach, 211th ECS meeting, May 2007.

[17] P. Chang, G.-S. Kim, K. Promislow, B. Wetton, Reduced dimensional computational models of polymer electrolyte membrane fuel cell stacks, Journal of Computational Physics 223 (2) (2007) 797-821. 
[18] P.C. Sui, S. Kumar, N. Djilali, Advanced computational tools for PEM fuel cell design. Part 2. Detailed experimental validation and parametric study, Journal of Power Sources 180 (1) (2008) 423-432.

[19] A.A. Shah, T.R. Ralph, F.C. Walsh, Modelling and simulation of the degradation of perfluorinated ion-exchange membranes in PEM fuel cells, Journal of the Electrochemical Society 156 (4) (2009) B465-B484.
[20] User Manual, CFD-ACE+, v. 2006, ESI-CFD, Huntsville, AL, 2006.

[21] P.C. Sui, N. Djilali, Analysis of coupled electron and mass transport in the gas diffusion layer of a PEM fuel cell, Journal of Power Sources 161 (1) (2006) 294-300.

[22] R.B. Bird, W.E. Stewart, E.N. Lightfoot, Transport Phenomena, 2nd ed., Wiley, New York, 2006. 